\title{
IMPORTÂNCIA DA RESSONÂNCIA MAGNÉTICA NO DIAGNÓSTICO E CONTROLE DA ESCLEROSE MÚLTIPLA: UM ESTUDO COM PACIENTES DA ASSOCIAÇÃO GOIANA DE ESCLEROSE MÚLTIPLA
}

\author{
Cristiene Costa CARNEIRO \\ Denise Sisterolli DINIZ \\ Francysdony Flávio Almeida CRUZ \\ Lee CHEN CHEN
}

\begin{abstract}
${ }^{\mathrm{a}}$ Mestre em biologia e doutoranda pela Universidade Federal de Goiás, profacristiene@ gmail.com
${ }^{b}$ Doutora em ciências da saúde e professora adjunto na Faculdade de Medicina da Universidade Federal de Goiás, dsd@brturbo.com.br

cEspecialista em docência do ensino superior e biomédico no banco de sangue Hemolabor, flávio_sma@ hotmail.com

${ }^{\mathrm{d}}$ Doutora em ciências e professora associada no Instituto de Ciências Biológicas da Universidade Federal de Goiás, chenleego@yahoo.com.br
\end{abstract}

Recebido em: 30/10/2013 - Aprovado em: 20/12/2013 - Disponibilizado em: 15/01/2014

\begin{abstract}
RESUMO: A esclerose múltipla é uma doença desmielinizante do sistema nervoso central (SNC) caracterizada por manifestar-se através de surtos que evoluem ao acaso e por causa de diagnóstico tardio. O caráter inespecífico dos sintomas é o principal responsável por dificultar o diagnóstico da doença. Neste trabalho, foi avaliada a contribuição da ressonância magnética no diagnóstico e controle da esclerose múltipla, bem como os dados clínicos e socioeconômicos de pacientes da Associação Goiana de Esclerose Múltipla. No total, foram entrevistados 16 pacientes da associação. Pelos resultados obtidos, observou-se predominância do sexo feminino e da etnia branca. A maioria dos associados têm idade acima de 40 anos. As condições sócio-econômicas dos pacientes entrevistados em geral são boas. Os sintomas de dormência, desequilíbrio/tonturas e fadiga predominaram como sintomas iniciais. Diante das primeiras manifestações clínicas, 37,5\% dos pacientes procuraram um neurologista de imediato. Na primeira consulta, $62,5 \%$ dos pacientes não tiveram a devida suspeita clínica. Essa mesma porcentagem foi observada entre os pacientes que receberam tratamento não específico antes de iniciarem o tratamento para esclerose múltipla. Todos os pacientes entrevistados (100\%) realizaram a ressonância magnética para complementar a suspeita clínica da doença. Quatro (4) pacientes (25\%) afirmaram já ter modificado o tratamento por causa de uma ressonância. As dificuldades financeiras de uma parcela relevante de pacientes $(37,5 \%)$ prejudicam a realização desse exame de forma regular. A maioria dos pacientes entrevistados $(43,75 \%)$ realiza a ressonância por controle a cada ano.
\end{abstract}

Palavras-chave: Esclerose múltipla. Ressonância magnética. Diagnóstico. Controle.

\begin{abstract}
Multiple sclerosis is a demyelinating disease of the central nervous system (CNS) characterized by manifest itself through of episodes which evolve randomized and because of late diagnosis. The nonspecific nature of the symptoms is the main responsible for the disease make diagnosis difficult. In this study, we evaluated the contribution of magnetic resonance imaging in the diagnosis and control of multiple sclerosis, as well as clinical and socioeconomic data of patients from Associação Goiana de Esclerose Múltipla. In total, 16 patients were interviewed in the association. From the results obtained, there was a predominance of females and caucasians. The majority of associated were aged over 40 years. In general, the socioeconomic conditions of the patients interviewed are good. Symptoms of numbness, imbalance / dizziness and fatigue predominated as the initial symptoms. With the appearance of the first clinical signs, $37.5 \%$ of the patients seeked a neurologist immediately. At first visit, $62.5 \%$ of patients had adequate clinical suspicion. This same percentage was observed among patients who received no specific treatment before starting treatment for multiple sclerosis. All interviewed patients $(100 \%)$ underwent magnetic resonance imaging to complement the clinical suspicion of the disease. Four (4) patients $(25 \%)$ said they had changed treatment because of a resonance. The financial difficulties of a relevant portion of patients (37.5\%) make it difficult to perform this test of form regular basis. Most of the interviewed patients (43.75\%) performs resonance by control the each year.
\end{abstract}

Key-words: Multiple sclerosis. Magnetic resonance. Diagnosis. Control. 


\section{INTRODUÇÃO}

Dentre as doenças desmielinizantes do Sistema Nervoso Central (SNC), a Esclerose Múltipla (EM) se destaca por sua diversidade de apresentações clínicas, o que torna seu diagnóstico desafiante para a maioria dos neurologistas (MINGUETTI, 2001; HUIJBREGTS, 2004).

Atualmente, é conhecida como uma doença inflamatória crônica auto-imune que provoca a destruição da bainha de mielina. Geralmente atinge indivíduos jovens adultos, na faixa etária entre 20 e 40 anos de idade, com predomínio no sexo feminino e etnia branca. Geograficamente apresenta maior incidência nas zonas temperadas e frias, situadas aproximadamente entre 40 e 60 graus de latitude norte e sul (SOUZA, 2000; MINGUETTI, 2001; HAUSSEN, 2005; LEITE et al., 2008).

Por serem inespecíficos e mais brandos no início da doença, os primeiros sintomas geralmente levam o paciente e os médicos não especialistas em neurologia, a confundir a EM com outras patologias (SOUZA, 2000; GIULIANE et al., 2005). Isso dificulta o diagnóstico precoce, essencial para um tratamento eficaz.

Além dos sintomas clínicos não específicos, outra dificuldade encontrada para o diagnóstico da doença é a inexistência de exames laboratoriais específicos (SOUZA, 2000).
Em 2001, McDonald et al. estabeleceram alguns critérios para facilitar o diagnóstico da EM. Apesar de não serem aceitos internacionalmente, eles estabelecem que a combinação dos sintomas clínicos, com investigações das lesões típicas de EM através de Ressonância Magnética ( $\mathrm{RM})$ cerebral e cervical, estudo dos potenciais evocados (EPE) e presença de bandas oligoclonais no Líquido Céfalo Raquidiano (LCR), pode fornecer evidências que levam a um diagnóstico mais seguro.

Em ordem cronológica, o primeiro método laboratorial usado com a finalidade de reforçar a suspeita clínica de EM foi a análise do LCR. Em 1960, pesquisadores reconheceram que a EM se associava a uma gamopatia oligoclonal do líquor, que implicava no aumento de imunoglobulina (IgG) (FARLOW; BORNIN, 1993). O EPE, que detecta lesões do nervo óptico, lesões medulares e lesões do tronco cerebral, passou a ser usado em pacientes com EM a partir de 1970. Mas essas lesões encontradas através do EPE nem sempre representam lesões de EM (HAUSSEN, 2005).

Com o surgimento das técnicas de neuroimagem, a partir dos anos setenta, foi possível estudar com maiores detalhes as lesões típicas de Esclerose Múltipla. A Tomografia Computadorizada não contribuiu muito para o diagnóstico da doença. Em contrapartida, a Ressonância Magnética tem demonstrado elevada sensibilidade para 
lesões na substância branca (ROLAK, 2003; RINCK, 2008).

Entretanto, a Ressonância Magnética não é capaz de diferenciar a Esclerose Múltipla de algumas patologias, como ocorre no caso da Encefalomielite Disseminada Aguda (EMDA). Isso ocorre porque as lesões da EM são idênticas às lesões de EMDA, com a única diferença que estas últimas não evoluem em surtos, como acontece na EM (OSBORN et al., 2004). Daí a importância da observação clínica do paciente e do controle evolutivo da doença através desse exame.

Além do diagnóstico diferencial, a Ressonância Magnética é utilizada no acompanhamento evolutivo da EM, fornecendo informações a respeito da disseminação ou não das lesões no tempo e no espaço (BAKSHI et al., 2004). Esse controle evolutivo é importante, porque indica se o tratamento está sendo eficiente em conter a evolução natural da EM em pacientes já diagnosticados com a doença (CALABRESI, 2004).

Desta forma, a RM apresenta grande importância na confirmação diagnóstica de casos clínicos suspeitos de EM, como também no controle evolutivo de pacientes já diagnosticados com a doença. Por isso, o objetivo do presente trabalho foi analisar a contribuição da RM no diagnóstico e controle da EM para os pacientes da Associação Goiana de Esclerose Múltipla (AGEM), bem como analisar o perfil clínico e socioeconômico desses pacientes, a fim de contribuir para um melhor entendimento das características epidemiológicas de pacientes goianos com EM.

\section{MATERIAIS E MÉTODOS}

Após aprovação do Comitê de Ética em Pesquisa da Universidade Federal de Goiás (UFG), sob protocolo número 052/2009, o presente estudo foi realizado com membros da Associação Goiana de Esclerose Múltipla, através da aplicação de um questionário (Apêndice $\mathrm{A}$ ) aos associados que concordaram em assinar o Termo de Consentimento Livre e Esclarecido (TCLE) (Apêndice B).

Todos os participantes da associação têm diagnóstico positivo da doença. Foram entrevistados 16 portadores de EM membros da AGEM durante duas reuniões realizadas nos dias 26 de Junho e 24 de Julho de 2009, na própria sede da Associação, localizada na rua J-44, Qd. 85 Lt. 07 do setor Jaó, GoiâniaGO/CEP: 74673-610.

Assim, a entrevista com pacientes da AGEM resultou numa pesquisa quantitativa e descritiva, e os dados coletados foram analisados utilizando parâmetros numéricos, representações gráficas, testes paramétricos e/ou não paramétricos. 


\section{RESULTADOS E DISCUSSÃO}

O presente estudo realizado na AGEM teve a participação de 16 voluntários, sendo $12(75 \%)$ do sexo feminino e quatro (4) $(25 \%)$ do sexo masculino, perfazendo uma relação de 3:1 (Gráfico 1). Tal resultado demonstra que houve predomínio do sexo feminino com relação ao masculino, o que é compatível com diversos estudos com pacientes portadores de EM (TOMAZ et al., 2005; SANTOS et al., 2007; MICHAEL, 2013).

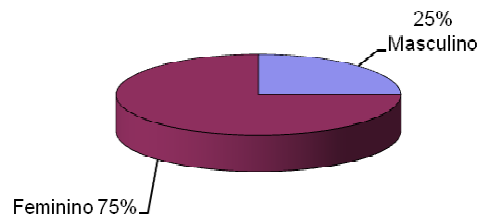

Gráfico 1 Distribuição dos pacientes da AGEM por sexo.

Em relação à distribuição por etnia, a maioria dos entrevistados eram brancos (15) $(93,75 \%)$ e apenas um (1) $(6,25 \%)$ era negro (dado em concordância com a literatura) (ARRUDA et al., 2001; SANTOS; YOKOTA; DIAS, 2007; MICHAEL, 2013) (Gráfico 2). Moreira et al. (2000) encontraram $5 \%$ de pacientes negros num estudo realizado em São Paulo, porcentagem muito semelhante à encontrada neste trabalho $(6,25 \%)$.

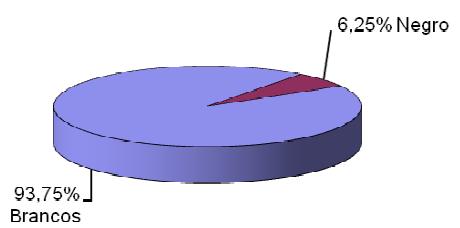

Gráfico 2 Distribuição dos pacientes da AGEM por etnia.

Quanto à idade, a EM é uma doença caracterizada por manifestar-se em adultos jovens, com idade entre 20 e 40 anos, ocorrendo com menor freqüência em crianças e adolescentes (3 a 7\%) e em indivíduos acima de 50 anos de idade (9\%) (GROSSMAN; YOUSEM， 2003). No presente estudo, não obtivemos casos de pacientes muito jovens, ou seja, antes dos 19 anos de idade, em contrapartida, houve predomínio de pacientes com idade acima dos $40 \operatorname{anos}(62,5 \%)$ e entre $20-39$ anos $(37,5 \%)$ (Gráfico

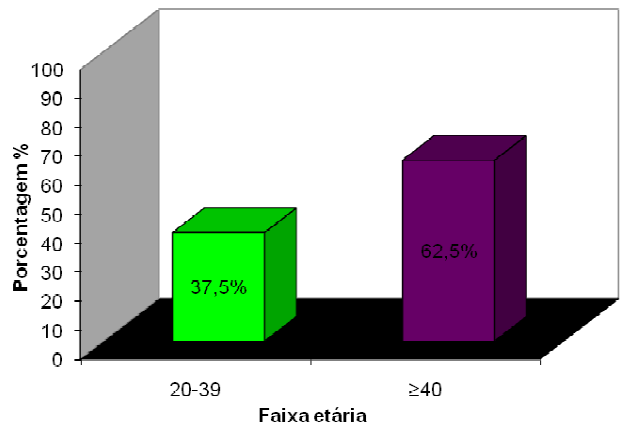

Gráfico 3 Faixa etária dos pacientes da AGEM 
No questionário aplicado a AGEM optamos por incluir uma questão a cerca da existência ou não de plano de saúde privado por parte dos associados, pois acreditamos que essa seja uma grande ferramenta para obtenção de diagnóstico e controle evolutivo da EM no Brasil, principalmente através da RM. Sendo assim, constatamos que dos 16 entrevistados, 12 tinham plano de saúde privado, Unimed (5) ou Ipasgo (7), e quatro (4) não possuíam auxílio desse tipo de plano (Tabela 1). Em se tratando de uma pequena amostra como a que utilizamos neste estudo, a porcentagem de pacientes desprovidos de plano de saúde foi estatisticamente significativa $(25 \%)(\mathrm{p}<0,05)$.

Tabela 1 Plano de saúde existente entre os pacientes da AGEM.

*Sistema único de Saúde.

A Constituição Federal de 1988, Capítulo VII da Ordem Social-Seção II, por meio do Artigo 196, assegura que “A saúde é direito de todos e dever do Estado“. A Lei Orgânica da Saúde (Lei 8.080), que define o modelo operacional do SUS, permite à iniciativa privada participar do SUS apenas em caráter complementar.

Pelo estabelecimento da lei todos devem ser bem atendidos pelo SUS. Todavia, a situação atual de saúde pública no Brasil não parece muito animadora. São filas freqüentes de pacientes nos serviços públicos de saúde; falta de leitos hospitalares para atender a demanda da população; dificuldade em se conseguir exames e procedimentos de alto custo; falta de medicamentos, principalmente os de alto custo; atraso no repasse dos pagamentos do Ministério da Saúde para os serviços conveniados, entre outras inúmeras situações. Toda esta complexa realidade do setor de saúde no Brasil revela o quanto a política social do país ainda está distante do modelo desejado.

Se nos voltarmos para a realidade dos portadores de EM de Goiânia, veremos que a situação deles não é muito diferente da apresentada no parágrafo anterior. Conforme exposto anteriormente, a EM é uma doença de difícil diagnóstico, que requer profissionais competentes e uma investigação clínica e laboratorial criteriosa. Para que isso ocorra

\begin{tabular}{l|c}
\hline Plano de Saúde & $\begin{array}{c}\text { Número de } \\
\text { pacientes }\end{array}$ \\
\hline Unimed & 5 \\
Ipasgo & \\
SUS ${ }^{*}$ & 4 \\
\hline Total & necessários \\
\hline \multicolumn{1}{c}{ alguns } \\
são exames \\
complementares, dos quais a RM é o mais \\
indicado (CALLEGARO et al., 2001; \\
VASCONCELOS; ALVARENGA, 2007). \\
alto custo, a RM não é disponibilizada em
\end{tabular}


muitos hospitais públicos de Goiânia e muitos serviços que disponibilizam o exame pelo SUS acabam desistindo do convênio devido a atrasos no repasse dos pagamentos e a baixos valores pagos pelo procedimento.

\section{Da mesma forma como Fragoso e} Peres (2007) sugeriram que os resultados obtidos em seu estudo poderiam não refletir a situação real sobre a prevalência da EM na cidade de Santos, face às dificuldades de acesso aos meios de diagnóstico da doença, sugerimos que em Goiânia, muitos casos de pacientes com suspeita clínica de EM ainda estão sem diagnóstico e sem receber tratamento específico.

Em relação às primeiras manifestações clínicas apresentadas pelos pacientes da AGEM, as mais comuns foram: dormências (62,5\%); desequilíbrio e tonturas $(43,75 \%)$; fadiga $(43,75 \%)$; alterações visuais $(37,5 \%)$; déficit motor (25\%); déficit de atenção e aprendizado (18,75\%); tremores $(6,25 \%)$; incontinência urinária $(6,25 \%)$; paralisia facial $(6,25 \%)$; alterações da linguagem $(6,25 \%)$; cefaléia $(6,25 \%)$ e choques na coluna e membros inferiores $(6,25 \%)$. Alguns pacientes tiveram dificuldade em relatá-las principalmente devido ao caráter inespecífico dessas manifestações.

Em três estudos realizados no Brasil, os sintomas sensitivos (dormências, formigamento, perda de sensibilidade etc) e cerebelares (desequilíbrio, tontura, vertigem etc) predominaram como sintomas iniciais, conforme encontramos em nosso trabalho. Todavia, foi observado que o número de casos de pacientes com apresentação clínica cerebelar foi maior do que aqueles com sintomas sensitivos: Arruda et al. (2001) obtiveram $63 \%$ e $53 \%$ de casos com sintoma cerebelar e sensitivo respectivamente, Tomaz et al. (2005) reportaram $60 \%$ e $43,3 \%$ e Gzresiuk (2006) relatou $40 \%$ e $20 \%$.

A fadiga, as alterações visuais (neurite óptica), déficit motor e de atenção e aprendizado, também estiveram entre os sintomas iniciais mais comuns relatados pelos pacientes da AGEM, e são encontrados com muita freqüência na literatura (ARRUDA et al., 2001; FIGUEIRA, 2007). Os sintomas de tremores, incontinência urinária, paralisia facial e choques na coluna e membros inferiores também são bastante comuns na EM, apesar de não terem apresentado elevada porcentagem em nosso estudo (6,25\%) (VASCONCELOS; ALVARENGA, 2007).

Dificuldade de articulação das palavras e cefaléia são freqüentemente associadas com outras patologias como Acidente Vascular Cerebral (AVC) e enxaqueca, o que prejudica o diagnóstico definitivo da EM de maneira precoce (CALLEGARO, 2001; FERREIRA; SILVA, 2003).

Com relação à expectativa de vida dos pacientes com EM, pode-se afirmar que ela 
depende de inúmeros fatores, dos quais se destacam a forma progressiva manifesta (se surto-remitente, primariamente-progressiva, secundariamente-progressiva ou progressivarecorrente), o diagnóstico precoce e o tratamento eficiente, com acompanhamento clínico e laboratorial criterioso (GROSSMAN; YOUSEM, 2003).

Em nosso estudo, a questão que se refere ao tempo de doença dos pacientes da AGEM mostrou que $37,5 \%$ deles haviam apresentado os primeiros sintomas entre zero (0) e quatro (4) anos atrás; $25 \%$ entre cinco (5) e nove (9) anos e 37,5\% há dez (10) anos ou mais. Também foi observado que a maioria dos pacientes entrevistados manifestou a doença entre 20 e 40 anos de idade. Portanto, esses dados são compatíveis com diversos estudos que relatam a cerca da idade na qual os primeiros sintomas da EM se manifestam (MOREIRA et al., 2000; FERREIRA et al., 2004). Por outro lado, foi observado que a expectativa de vida dos portadores de EM tem aumentado.

Diante de qualquer sintoma possivelmente relacionado à EM a conduta mais adequada do paciente deveria ser a procura de um médico especialista na doença, o neurologista (SOUZA, 2000). Entretanto, por desconhecimento da doença, ou mesmo pelo caráter inespecífico dos sintomas, o paciente acaba recorrendo a outros médicos e dificultando o diagnóstico definitivo da EM.
Dos 16 entrevistados em nosso trabalho, seis (6) $(37,5 \%)$ procuraram um neurologista de imediato, três (3) $(18,75 \%)$ o procuraram por indicação de amigos ou familiares, quatro (4) (25\%) por indicação de outros médicos (ou seja, consultaram outros médicos antes de passarem pelo neurologista), dois (2) (12,5\%) por suspeitarem de AVC e um (1) $(6,25 \%)$ por causa do resultado de uma RM de crânio que outro médico havia lhe prescrito.

Se o médico neurologista suspeitar da doença ainda na primeira consulta, o paciente tem grande chance de receber diagnóstico precoce e tratamento específico, podendo até evitar seqüelas graves, decorrentes de surtos posteriores e mais agressivos (FIGUEIRA, 2007). No presente trabalho, $37,5 \%$ dos entrevistados relataram que o neurologista suspeitou da doença na primeira consulta, enquanto $62,5 \%$ não tiveram essa suspeita. Além disso, 62,5\% dos pacientes chegaram a realizar tratamento não específico até receber diagnóstico definitivo de EM, o que pode ter contribuído para que uma parcela considerável desses pacientes (18,7\%) apresentasse seqüelas da doença antes de iniciarem o tratamento específico. Apesar de nem todos terem apresentado sequielas nos surtos iniciais, muitos pacientes $(31,25 \%)$ demonstraram-se insatisfeitos com o seu diagnóstico ao considerá-lo tardio.

Sendo assim, concluímos que o diagnóstico clínico da EM não representa uma 
tarefa fácil para a maioria dos neurologistas, pelo contrário, representa um grande desafio que requer principalmente tempo com o paciente no consultório. Diversos autores declaram que a inespecificidade dos sintomas é o principal responsável por tornar o diagnóstico da EM tão difícil (GROSSMAN; YOUSEM, 2003; VASCONCELOS; ALVARENGA, 2007). Todavia, para que o neurologista estabeleça o diagnóstico final da EM com segurança, torna-se imprescindível a solicitação de exames laboratoriais que complementem a suspeita clínica. De acordo com os critérios de McDonald et al. (2001) essa investigação laboratorial se baseia principalmente na $\mathrm{RM}$ de crânio e coluna cervical, análise do LCR e dos potenciais evocados.

Todos os pacientes entrevistados (100\%) responderam que a RM de Crânio foi solicitada pelo neurologista no momento em que houve a suspeita clínica da doença. Além da RM, o exame do LCR foi solicitado em $81,25 \%$ dos casos e o estudo dos potenciais evocados em $56,5 \%$ deles.

Tais resultados estão em concordância com um estudo realizado na cidade de Cuiabá, no qual todos os pacientes com diagnóstico de EM haviam realizado RM de Crânio diante da suspeita clínica e $55 \%$ haviam realizado o exame do LCR (GRZESIUK, 2006). Os estudos dos potenciais evocados solicitados em $56,5 \%$ dos pacientes do presente estudo também se aproximam dos 44,5\% dos pacientes avaliados através desse exame na Secretaria de Estado da Saúde de Minas Gerais (SANTOS; YOKOTA; DIAS, 2007).

Em relação à mudança ou não de medicamentos por causa do resultado de uma RM, dois pacientes (12,5\%) não souberam responder a questão, quatro (25\%) já tiveram que mudar os medicamentos e 10 (62,5\%) responderam que não.

Esses resultados demonstram que a porcentagem de pacientes (25\%) que em algum momento mudaram os medicamentos por causa de uma RM foi relevante e reflete mais uma aplicação desse exame na doença: o monitoramento do tratamento.

Entre as maiores dificuldades encontradas pelos pacientes da AGEM para realizarem a RM destacam-se as dificuldades financeiras $(37,5 \%)$, as limitações de alguns planos de saúde $(12,5 \%)$, o medo de ficar em lugar fechado (12,5\%), a demora do exame $(6,25 \%)$ e especialmente a insuficiência de serviços públicos que ofereçam a RM $(25 \%)$. Também houve uma parcela significativa de pacientes que declararam não ter dificuldades para realizar o exame $(43,75 \%)$.

Pelos resultados obtidos, pôde-se observar que a maioria dos associados não apresenta dificuldades para realizar a RM $(43,75 \%)$. Entretanto, diante dos pacientes que responderam possuir dificuldades 
financeiras $(37,5 \%)$ e insuficiência de serviços públicos que ofereçam esse exame (25\%), pode-se afirmar que ele ainda é um método de diagnóstico e controle para a EM de difícil acesso e pouco disponível para a população goiana em geral.

Em relação à freqüência com que os pacientes da AGEM realizam a RM, observase que a maioria faz o exame uma vez por ano $(43,75 \%)$ ou sem regularidade $(18,75 \%)$, enquanto $12,5 \%$ o fazem quando apresentam novos surtos, $12,5 \%$ a cada seis meses, $6,25 \%$ a cada três meses e 6,25\% a cada dois anos. Observa-se, portanto, que nem todos os pacientes entrevistados realizam a RM por controle dentro do intervalo ideal, que é de 6 meses a 1 ano.

Portanto, o presente estudo espera contribuir para um melhor entendimento das características epidemiológicas de pacientes goianos com EM, e ainda mobilizar órgãos competentes que sejam capazes de oferecer gratuitamente o exame de RM para toda a população, visto que o diagnóstico e o controle evolutivo da EM dependem fundamentalmente desse exame.

\section{AGRADECIMENTOS}

Nós agradecemos a todos os pacientes da Associação Goiana de Esclerose Múltipla que aceitaram participar desse estudo, em especial, a presidente da AGEM, Eduarda Assis de Albuquerque.

\section{REFERÊNCIAS}

ARRUDA W.O.; SCOLA R.H.; TEIVE H.A.G.; WERNECK L.C. Multiple Sclerosis: Report on 200 cases from Curitiba, Southern Brasil and comparison with other Brazilian series. Arquivos de Neuropsiquiatria, v. 59, n. 1, p. 165-170, 2001.

BAKSHI R.; HUTTON G.J.; MILLER J.R.; RADUE E.W. The use of magnetic resonance imaging in diagnosis and long-term management of multiple sclerosis. Neurology, v. 63, n. 11, p. 83-91, 2004.

Disponível em:<http://ovidsp.tx.ovid.com/spa /ovidweb.cgi.pdf\&filename $=$ The + use + of + ma gnetic+resonance+imaging+in+the+diagnosis + and+longterm+management + of + multiple $+\mathrm{sc}$ lerosis.>.

CALLEGARO D. Diagnóstico e Tratamento da Esclerose Múltipla. AMBCFM: Projeto Diretrizes, v. 1, n. 2, p. 1-10, 2001.

CALABRESI, P.A. Diagnosis and Management of Multiple Sclerosis. American Family Physician, v.15, n. 10, p. 1-14, 2004. Disponível em: < http://www.aafp.org/afp/20041115/1935.pdf $>$.

FERREIRA K.S.; SILVA W.F. Eficácia do tratamento terapéutico ocupacional na esclerose múltipla. 2003. $46 \mathrm{f}$. Trabalho de Conclusão de Curso em Fisioterapia Universidade Católica de Goiás, Goiânia, 2003.

FERREIRA M.L.B. et al. Epidemiologia de 118 casos de Esclerose Múltipla com seguimento de 15 anos no Centro de Referência do Hospital da Restauração de Pernambuco. Arquivos de Neuropsiquiatria, v. 62, p. 1027-1032, 2004. 
FIGUEIRA F. Síndromes Clinicamente Isoladas. Abbot, v. 1, n. 3, p. 1-5, 2007.

FRAGOSO Y.D.; PERES M. Prevalence of multiple sclerosis in city of Santos, SP. Revista Brasileira de Epidemiologia, v. 10, n. 4, p. 479-482, 2007.

GIULIANE, I.M.F. et al. Esclerose Múltipla: Conscientização e luta pelo reconhecimento de direitos. Estudos Jurídicos Cidadania e Direitos, v.2, n.2, p. 9-10, 2005. Disponível em:<http://trt15.gov.br/boletim/estudos_juridic os2005_2.pdf $>$.

GROSSMAN R.I.; YOUSEM D.M. Primary Demyelinating Disease: Multiple Sclerosis. In: Neuroradiology: the requisites. 2. ed. Elsevier: Mosby, p. 332-347, 2003.

HAUSSEN S.R. Esclerose Múltipla. In: PORTO CC. Semiologia Médica. $5^{\mathrm{a}}$ ed. Rio de Janeiro: Guanabara Koogan, p. 1171-1173, 2005.

HUIJBREGTS S.C.J.; KALKERS N.F.; SONNEVILLE L.M. ; GROOT V.; REULING I.E. ; POLMAN C.H. Differences in cognitive impairment of relapsing remitting, secondary, and primary progressive MS. Neurology, v. 63, n. 2, p. 335-339, 2004.

LEITE C.C.; AMARO JÚNIOR E.; LUCATO L.T. Doenças da substância branca adquiridas ou desmielinizantes: Esclerose Múltipla. In: NEURORRADIOLOGIA: Diagnóstico por imagem das alterações encefálicas. 1. ed. Rio de Janeiro: Guanabara Koogan, 2008. Cap. 11.

MCDONALD W.I.; COMPSTON A.; EDAN G.; GOODKIN D.; HARTUNG H.P.;

LUBLIN F.D. et al. Recommended Diagnosis Criteria for Multiple Sclerosis: Guidelines from the International Panel on the Diagnosis of Multiple Sclerosis. Annals of Neurology, v. 50, n. 1, p. 121-127, 2001.

MICHAEL J.O. Epidemiology and clinical features of multiple sclerosis in adults. Uptodate, v. 21, p. 1689-1710, 2013.
MINGUETTI G. Ressonância Magnética na Esclerose Múltipla. Arquivos de Neuropsiquiatria, v. 59, n. 3-A, p. 563-569, 2001.

MOREIRA M.A.; FELIPE E.; MENDES M.F.; TILBERY C.P. Esclerose Múltipla: estudo descritivo de suas formas clínicas em 302 casos. Arquivos de Neuropsiquiatria, v. 58, p. 460-466, 2000.

OSBORN A.G et al. Multiple Sclerosis and ADEM. In: Diagnostic Imaging BRAIN. 1.ed. Canadá: Amyrsis, p. 875-881, 2004.

RINCK P.A. A short history of magnetic resonance imaging. Spectroscopy Europe, v. 20, n. 1, p. 500-5003, 2008.

SANTOS E.C, YOKOTA M.; DIAS N.F.R. Esclerose Múltipla: Estudo com pacientes com a forma surto-remissão cadastrados na Secretaria de Estado da Saúde de Minas Gerais. Arquivos de Neuropsiquiatria, v. 65, n. 3-B, p. 885-888, 2007.

SOUZA S.E.M. Esclerose Múltipla. In: Tratamento das doenças neurológicas. 1. ed. Rio de Janeiro: Guanabara Koogan, Cap. 184, 2000.

TOMAZ A.; BORGES F.N.; GANANÇA C.F.; CAMPOS C.A.H.; TILBERY C.P. Sinais e sintomas associados a alterações otoneurológicas diagnosticadas ao exame vestibular computadorizado em pacientes com esclerose múltipla. Arquivos de Neuropsiquiatria, v. 63, n. 3-B, p. 837-842, 2005.

VASCONCELOS C.; ALVARENGA M. Investigação Diagnóstica. Abbot, v. 2, p. 1-5, 2007. 


\section{APÊNDICE A:}

1- Nome:

2- Sexo:
( ) Masculino
( ) Feminino

3- Etnia:

( ) Mongolóide ou amarela

( ) Caucasóide ou branca

( ) Negróide ou negra

4- Idade:

( ) De 0 a 19 anos

( ) De 20 a 39

( ) Acima de 40

5- Você tem plano de saúde?

( ) Sim. Qual?

( ) Não

6- Você se lembra de quais foram os seus primeiros sintomas de Esclerose Múltipla?
( ) Visão dupla
( ) Dormências
( ) Desequilíbrio
( ) Fadiga
( ) Tremores
( ) Dificuldade de movimentação
( ) Outros: 
7- Há quanto tempo surgiram os primeiros sintomas antes mesmo da suspeita diagnóstica de Esclerose Múltipla?

( ) Há menos de 6 meses;

( ) De 6 meses a 1 ano;

( ) Há mais de 1 ano.

8- O que levou você a procurar um neurologista?

9- O médico neurologista suspeitou da doença ainda na primeira consulta?
( ) $\operatorname{Sim}$
( ) Não

10-Quando o seu médico suspeitou da doença, ele lhe indicou, entre outros exames, a Ressonância Magnética de Crânio?
( ) $\operatorname{Sim}$
( ) Não

11-A suspeita clínica de Esclerose Múltipla foi confirmada após análise de qual exame?

( ) Potencial Evocado;

( ) Análises Clínicas;

( ) Ressonância Magnética; ( ) Outros:

12-Você chegou a realizar algum tratamento não específico até receber o diagnóstico da doença?
( ) $\operatorname{Sim}$
( ) Não

13-Quando você iniciou o tratamento específico para Esclerose Múltipla, já apresentava seqüelas da doença?
( ) $\mathrm{Sim}$
( ) Não

14- Você considera seu diagnóstico precoce ou tardio?
( ) Precoce
( ) Tardio 
15- Alguma vez, você já teve que mudar os medicamentos por causa do resultado de uma Ressonância Magnética?
( ) Sim
( ) Não

16-Há quanto tempo trata a Esclerose Múltipla?

( ) Há menos de 6 meses;

( ) De 6 meses a 1 ano;

( ) Há mais de 1 ano

17-Quais são suas maiores dificuldades para realizar a Ressonância Magnética?

( ) Financeiras;

( ) Limitações de alguns planos de saúde;

( ) Medo de lugar fechado;

( ) Insuficiência de serviços públicos que ofereçam RM ;

( ) Não tenho dificuldades;

( ) Outras:

18-Com qual freqüência você faz a Ressonância Magnética?

( ) A cada mês;

( ) A cada 3 meses;

( ) A cada semestre;

( ) A cada ano;

( ) Quando apresenta novos sintomas;

( ) Sem regularidade

( ) Outros: 


\section{APÊNDICE B:}

\section{TERMO DE CONSENTIMENTO LIVRE E ESCLARECIDO}

Você está sendo convidado(a) para participar, como voluntário, de uma pesquisa. Após receber os esclarecimentos e informações a seguir, caso decida participar da pesquisa, assine, por favor, ao final deste documento. Em caso de recusa, você não será penalizado(a) de forma alguma. Em caso de dúvida, você pode procurar o Comitê de Ética em Pesquisa da Universidade Federal de Goiás, pelo telefone 3521-1075 ou 3521-1076.

\section{Informações sobre a pesquisa:}

Este questionário faz parte de um estudo acadêmico sobre a Importância da Ressonância Magnética no diagnóstico e controle da Esclerose Múltipla. Os objetivos gerais da pesquisa incluem a análise da contribuição da Ressonância Magnética no diagnóstico e controle da doença, e a avaliação das condições gerais dos portadores de EM.

Este estudo não acarretará riscos aos indivíduos participantes, pois o questionário a ser aplicado terá finalidade de obter informações para um estudo acadêmico, com a garantia do absoluto sigilo dos dados pessoais desses participantes. O presente estudo não fornece benefícios imediatos.

\section{Pesquisador Responsável}

\section{CONSENTIMENTO DA PARTICIPAÇÃO DA PESSOA COMO SUJEITO}

- A garantia de receber todos os esclarecimentos sobre as perguntas do questionário antes e durante o transcurso da pesquisa, podendo afastar-me em qualquer momento se assim o desejar, bem como estar assegurado o absoluto sigilo das informações obtidas.

- A segurança plena que não serei identificado(a) mantendo o caráter oficial da informação, assim como, estar assegurado(a) que a pesquisa não acarretará nenhum prejuízo individual ou coletivo.

- A segurança que não terei nenhum tipo de despesa material ou financeira durante o desenvolvimento da pesquisa, bem como, esta pesquisa não causará nenhum tipo de risco, dano físico ou mesmo constrangimento moral ou étnico. 
- A garantia de que toda e qualquer responsabilidade nas diferentes fases da pesquisa é dos pesquisadores, bem como, fica assegurado que independente dos resultados obtidos, o estudo poderá ser divulgado em órgãos de divulgação científica.

- Garantia de que todo material resultante será utilizado exclusivamente para construção da pesquisa e ficará sobre a guarda dos pesquisadores, durante um período de cinco (5) anos, podendo ser requisitado pelo entrevistado em qualquer momento. Ao término desse período, esse material será picotado e incinerado.

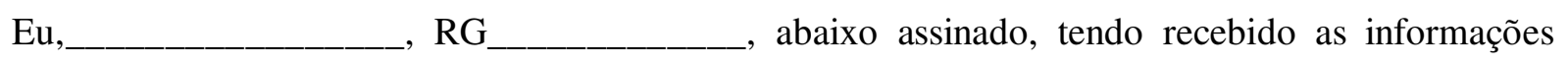
acima e ciente dos meus direitos relacionados, concordo em participar da pesquisa.

Tenho ciência do exposto acima e desejo participar da pesquisa.

Goiânia/GO,______e__________________

Assinatura do entrevistado

Impressão dactiloscópica 